\title{
Modeling of Consumers' Preferences for Regular Coffee Samples and Its Application to Product Design
}

\author{
Osamu TominagA, ${ }^{1}$ Fumio ITo, ${ }^{1}$ Taizo HANAI, ${ }^{2 *}$ Hiroyuki HondA ${ }^{2}$ and Takeshi KobaYASHI ${ }^{2 * *}$

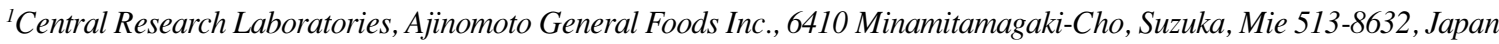 \\ ${ }^{2}$ Department of Biotechnology, School of Engineering, Nagoya University, Furo-cho, Chikusa-ku, Nagoya, aichi 464-8603, Japan
}

Received March 4, 2002; Accepted May 30, 2002

\begin{abstract}
A large-scale consumer test was made seeking preferences for regular coffee (RC). Based on preferences for 12 RC samples with various blend ratios of coffee beans, panels were divided into four preference clusters. Then, $88 \mathrm{RC}$ samples were prepared and preferences against them were tested for clustered panels. To predict preference scores for each cluster, highly accurate models were constructed by applying a fuzzy neural network. We then conducted reverse estimation for optimum preference blends on each cluster by applying a genetic algorithm. The RC samples of optimum preference blends identified above were prepared and preference tests were again performed for the same panels. Those samples showed good preference scores and good agreement with predictions by models for each cluster. Consequently, this approach, consisting of consumer clustering and modeling for each cluster, provides an excellent tool for the rapid and efficient development of coffee products.
\end{abstract}

Keywords: food engineering, preference modeling, coffee, genetic algorithm, fuzzy neural network, consumer clustering

To contribute to the establishment of an affluent society and comfortable consumer life, it is necessary to efficiently develop new products which meet consumers' preferences. For this purpose, the "Kansei engineering" (or sensory engineering) approach has been studied in recent years (Nagamachi, 1992; Sagara, 1998). In product design based on Kansei engineering, analysis and understanding of consumer preferences is very important, so much effort has been spent on marketing research. Consumer preferences are obviously diversified and many preference clusters exist. To satisfy cluster preferences, various products based on the same food have been produced and distributed on the market, e.g., more than 800 instant noodle products and 200 canned coffee products. Recent marketing activities divide consumers into several groups by their preference patterns for products and apply appropriate marketing methods to each cluster (Katahira, 1987). In product development, it is also important to understand the structure of consumer preference clusters and to know respective cluster characteristics, resulting in adjusted product quality, which meets the target cluster preferences.

Recent progress in knowledge information engineering has made it possible to analyze some complex relations. Consumer preference or behavior has been analyzed by an artificial neural network (ANN) (Nishio, 1994; Hamuro, 1997). However, it is difficult to elucidate explicitly causal relationships acquired in the ANN model. A fuzzy neural network (FNN), which has an ANN structure, can elucidate these relationships as a form of fuzzy logic (Furuhashi, 1993). We applied FNN to control the Ginjo sake mashing process (Hanai et al., 1997a; 1998d; Honda

*Present Address: Laboratory for Applied Biological Regulation Technology, School of Bioresource and Bioenvironmental Science, Kyushu University, 610-1 Hakozaki, Higashi-ku, Fukuoka 812-8581

**To whom correspondence should be addressed.

E-mail: takeshi@nubio.nagoya-u.ac.jp et al., 1998), to make models for activated sludge processes, for the quality of interior or exterior tile, and for the quality of beer or Ginjo sake (Hanai et al., 1998a; 1998b; 1998c; Noguchi et al., 1999; Tomida et al., 1999; 2001; Yoshikawa et al., 2001), to predict the motif in MHC class II binding peptides and the occurrence of red tide (Noguchi et al., 2001; Nagamori et al., 2001), and to assess Alzheimer-type dementia (Hibino et al., 2001). For regular coffee (RC), highly accurate sensory modeling to predict flavor and taste is indispensable in designing RC products reasonably. We constructed an FNN model (forward FNN) to predict the sensory score judged by professional coffee tasters for various blend ratios of coffee beans (Hanai et al., 1997b; Tominaga et al., 2002a). We also proposed a reliable method to determine the blend ratio of coffee with a target flavor and taste, which is mathematically the reverse calculation by applying a genetic algorithm with multi-objective ranking strategy and reliability index (RIGA) (Tominaga et al., 2002b). Our previous studies indicated that application of forward FNN modeling and the RIGA approach on consumer preference data would be very beneficial for development of a product which meets consumer preferences.

The present study involved a large-scale consumer test, grouped consumers by preference for coffee products, and built forward FNN models to predict preference for RC samples from the blend of coffee beans for each preference cluster. We then conducted reverse estimation for optimum preference blends on each cluster by RIGA. We also validated the performance of optimized blending samples.

\section{Materials and Methods}

Consumer tests A three-step consumer test was implemented. The first step was to divide consumer panels into four preference clusters; the second step was to build a prediction model for 
each preference cluster that was clarified by analysis in the first step; and the third step was to check preference of recommended blends that were reverse-estimated using the prediction model.

Clustering test Three representative coffee beans, the Colombian bean, Brazilian bean, and Indonesian bean, were used for blending in this paper. Each type of bean was roasted at one of three levels: light roast, medium roast, or dark roast. The nine kinds of single-bean regular coffee and three kinds of blended coffee $(1 / 3,1 / 3,1 / 3$ Blend) shown in Fig. 1 were ground to the same size ( $750 \mu \mathrm{m}$ average), and $90 \mathrm{~g}$ of those samples were packed in aluminum pouches with $\mathrm{N}_{2}$ gas to make RC samples. Consumer panelists $(n=650)$ were selected from among housewives living in the Tokyo and Osaka areas, aged between 20 and 55, who drink three or more cups of RC per week. Twelve RC samples were sent to panelists' homes with questionnaire sheets,

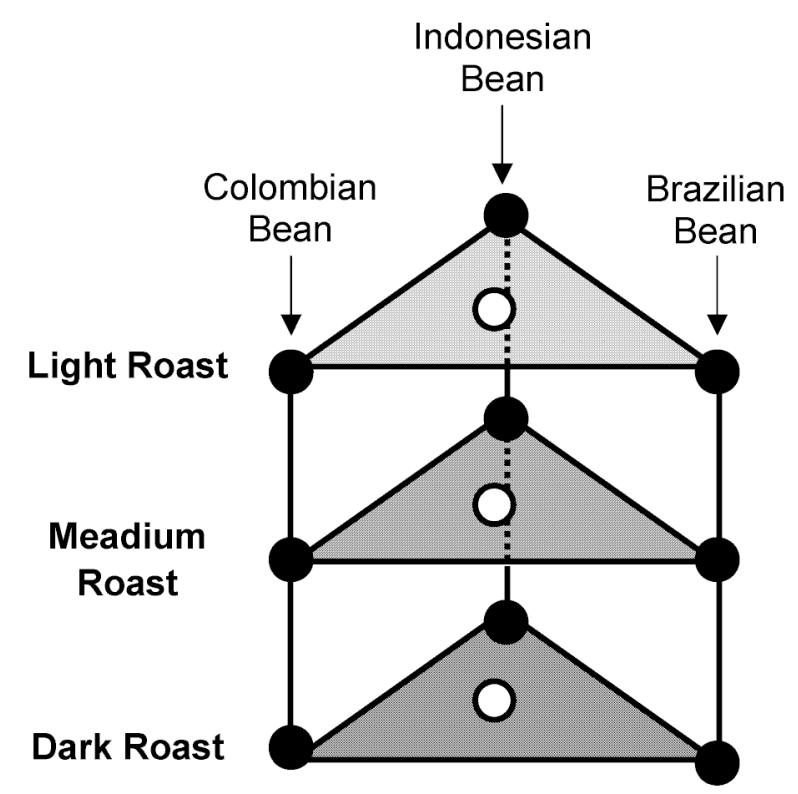

Fig. 1. Twelve kinds of regular coffee samples were used for clustering tests. Triangles show the 3-D graph indicating blend ratio. They consist of 9 single-bean blends made by roasting 3 degrees of roast color for beans of 3 origins $(\bullet)$, and 3 blended samples $(\bigcirc)$.

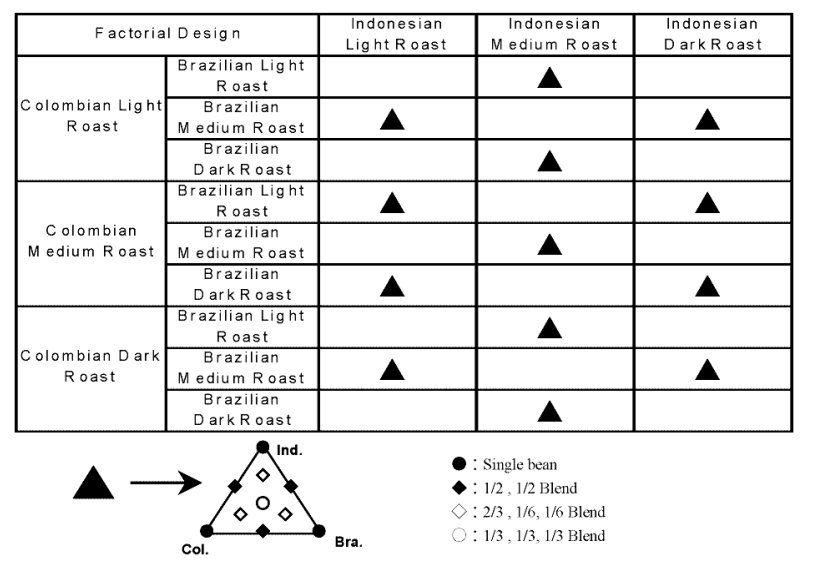

Fig. 2. Eighty-eight kinds of regular coffee samples were used for modeling tests. Combinations of 3 single-beans were selected by factorial design from the 9 single-beans shown in Fig. 1. Each combination marked by a solid triangle in this figure consists of 3 single-beans and 7 of their blends. and were tasted by the panelists during an 8 -week period. The panelists estimated their preferences for each RC sample using a 9-point scale in which a score of 1 indicated extreme dislike and a score of 9 indicated highest preference. After all evaluations, questionnaires were collected and all response data were used for clustering of consumer panels into four clusters.

Modeling test Thirteen combinations of the three beans shown in Fig. 2 were selected and 10 blends, including a single bean without blending in each combination, were provided. Blend ratios of these $\mathrm{RC}$ samples were determined according to factorial design. Consequently, $88 \mathrm{RC}$ samples with independent blend ratios were subjected to assessment against clustered panels. The same consumer panels in the clustering test were asked to evaluate a part of these 88 RC samples; finally, panelists $(n=500)$ whose preference clusters had been identified were employed for the modeling test. Tasting at home was carried out as described above. Questionnaires were collected to be input into a database. Then, models to predict preference scores for these RC samples were built for all preference clusters.

Validation test The same consumer panelists $(n=500)$ used in the clustering test were employed for the validation test. Four blended RC samples were prepared with blend ratios estimated to be the optimum ones for respective clusters by reverse calculation using RIGA. In this test, a panelist tasted one sample corresponding to her preference cluster. For example, sample 1, which was estimated to be the optimum blend ratio for cluster 1 preferences, was sent to the homes of panelists whose preference cluster was identified as cluster 1 . Then, preferences were tested as described above.

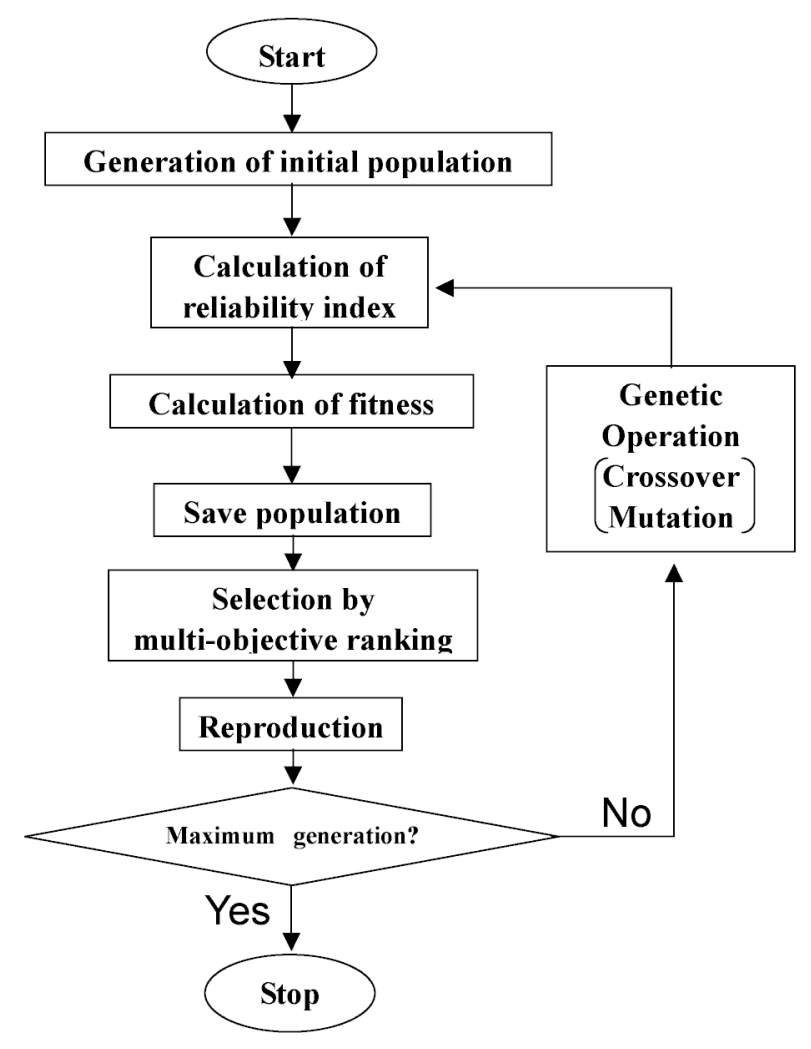

Fig. 3. Flow-chart of the RIGA operation program. 


\section{Analysis of data and modeling}

Preference cluster Cluster analysis was used for the clustering test data. Prior to this analysis, preference scores were standardized for each panel so that the mean value became 0 and the standard deviation value became 1 . The $\mathrm{k}$-means cluster analysis was executed based on procedures of FASTCLUS statistical software, the SAS ${ }^{\circledR}$ system (SAS Institute Inc., Carry, NC).

Preference model The mean preference score of each RC sample was calculated from the scores of panelists in each preference cluster in order to predict the preference score of the 88 RC samples. Type-I FNN models (Horikawa et al., 1991; Hanai et al., 1997a) were built for each preference cluster. Blend ratios of nine single beans consisting of three bean types and three roasting levels were used as input variables. Data of thirteen RC samples, which were randomly selected from among $39 \mathrm{RC}$ samples of the $2 / 3: 1 / 6: 1 / 6$ blend ratio as indicated in Fig. 2, were used for model evaluation; the remaining data of 75 samples were used for learning.

Reverse estimation To estimate optimum RC blends with maximum preference on each preference cluster, the RIGA proposed by us was used; both the multi-objective ranking selection

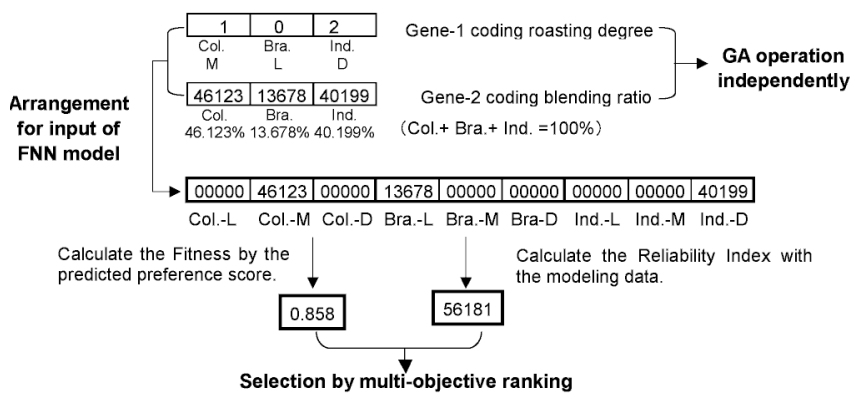

Fig. 4. Explanation of coding method of the blend ratio to genes and calculation method of fitness and RI. Abbreviations Col, Bra, and Ind indicate Colombian, Brazilian, and Indonesian beans, respectively; L, M, and D show the roast grades light, medium, and dark, respectively. and the reliability index $(R I)$ are incorporated in that RIGA (Hanai et al., 1998d; Tominaga et al., 2002b). The RIGA flow chart is shown in Fig. 3. Here, the new coding method was proposed to search real blend ratios efficiently, as shown in Fig. 4. We prepared two genes in each individual: one for providing the roasting degree of each bean origin, and the other for providing the blend ratio. Coding of the blend ratio was limited so that the sum of the blend ratio of the three beans equaled $100 \%$. Genes were operated independently during crossover and mutation. Fitness of individual $n(n=1,2, \ldots, N)$ was calculated by Eq. (1):

$$
\text { fitness }_{n}=1-\text { diff }_{n}^{2}
$$

where $N$ is the number of individuals (100 in the present study) and diff $_{n}$ indicates the difference between the desired preference value and the estimated preference value in each individual calculated by the forward FNN model. To determine the desired preference value, the maximum value of standardized preference score within all space for searching was calculated, that was then set as the target value. The $R I$ values of the individuals were calculated by Eq. (2) in this study:

$$
R I=-\frac{1}{3} \sum_{k=1}^{3} \frac{\log \text { error }_{k}^{2}}{r_{k}}
$$

In Eq. (2), $r_{k}$, indicates Euclidean distance between the point of interest and the $k$-th nearest data, and error ${ }_{k}$ indicates the learning error of the $k$-th nearest data on the preference score. The method to adjust the sum of the blend ratio to $100 \%$, the reproduction method, and other parameter settings were identical to our previous study (Tominaga et al., 2002b).

\section{Results and Discussion}

To roughly divide the consumer panelists into several clusters, we used 12 typical RC samples which were provided based on previous market-research experience acquired through Ajinomoto General Foods, Inc. Results of cluster analysis using the preference data of 650 consumers suggested that consumers were best divided into 4 preference clusters. By increasing the cluster

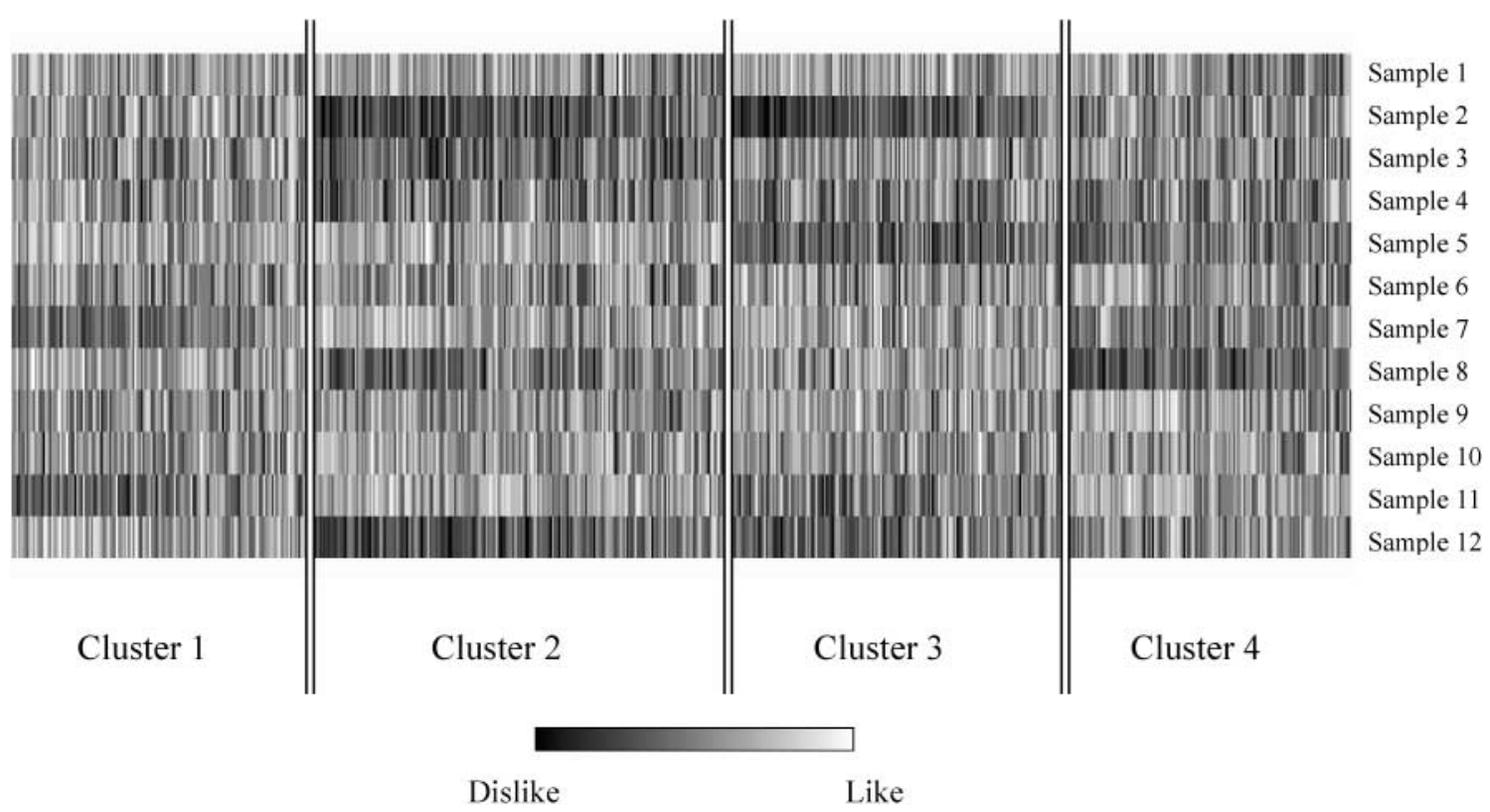

Fig. 5. Preference scores of each panelist and each sample on clustering tests. Panelists are sorted by distance calculated by $k$-means cluster analysis. 


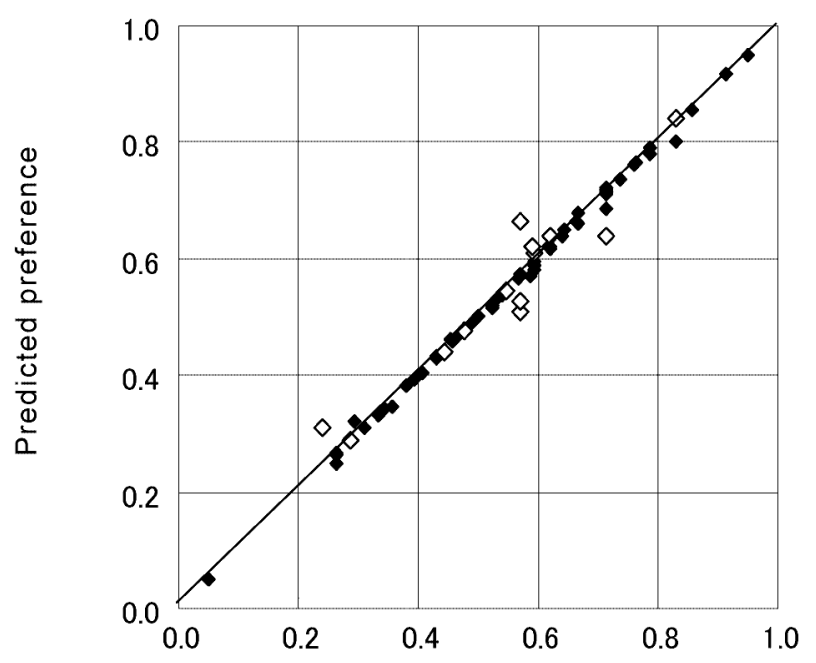

Actual preference

Fig. 6. Relationship between actual and predicted preference on the FNN model for cluster 1 . Closed and open rhombuses indicate learning and evaluation data, respectively.

number, preference patterns in the same cluster showed greatly improved consistency. However, it is likely that data reliability for formulating a preference model is lowered by the fewer number data points in each preference cluster.

Figure 5 illustrates the preference score for respective RC samples by each correspondent classified by four clusters. It is clear that consumer preference in each cluster showed a similar pattern, and patterns of different clusters were not the same. Furthermore, commonly preferred samples or disliked samples in each cluster were also observed. For example, cluster 1 panelists preferred samples 5,8 , and 12 , but not samples 7 or 11 . This knowledge of consumers' preference characteristics can be viewed as extremely advantageous and important for marketing activity.

In the next step, the modeling test was performed using 500

Table 1. Preference scores averaged by each cluster on modeling test data.

\begin{tabular}{|c|c|c|c|}
\hline & \multicolumn{2}{|c|}{ Average of preference score on each sample } & \multirow{2}{*}{$\begin{array}{l}\text { Standard deviation } \\
\text { of preference score }\end{array}$} \\
\hline & $\begin{array}{c}\text { Maximum among } 88 \\
\text { samples }\end{array}$ & $\begin{array}{c}\text { Minimum among } 88 \\
\text { samples }\end{array}$ & \\
\hline Cluster 1 & 6.46 & 4.88 & 1.53 \\
\hline Cluster 2 & 6.42 & 4.06 & 1.56 \\
\hline Cluster 3 & 6.38 & 4.72 & 1.54 \\
\hline Cluster 4 & 6.67 & 4.92 & 1.52 \\
\hline
\end{tabular}

consumer panelists among the 650 whose preference clusters had been confirmed. The range of the average and the standard deviation of preference scores toward the $88 \mathrm{RC}$ samples in respective clusters are shown in Table 1.

An FNN model was constructed to predict the preference score in each cluster, using data in which blend ratios of RC samples were input. The typical relationship between the predicted value and the actual one for cluster 1 preference is shown in Fig. 6. Correlation coefficients, mean absolute errors, and maximum absolute errors between predicted and actual values in each preference cluster are shown in Table 2. These results indicate the very high accuracy of the constructed FNN models since high values of correlation coefficients and significantly smaller values of maximum absolute error were obtained compared with the variances in preference score data in Table 1.

As the third step, optimum preference blending was sought in each cluster. Solutions expressing maximum preference scores were obtained by reverse calculation with RIGA using the forward FNN model and are shown in Table 3. The blending solution for each target cluster was estimated to be the most preferable to the target cluster and actually showed the highest score among the $88 \mathrm{RC}$ samples, indicating successful reverse calculation by RIGA.

Fitness and $R I$ values for each blend determined were larger than -0.3 and larger than 70 , respectively. If the fitness value is large, the determined blend shows the output from the forward FNN model close to the maximum preference score. If the $R I$ value is large, then the accuracy of the blend is high since the blend is located at the dense and reliable area. The blend is selected by the sum of fitness and $R I$-rank. In all space for searching, $R I$ values ranged from 300 (maximum) to 6 (minimum). When an exhaustive search was done as described in the previous research (Tominaga et al., 2002b), the RI solution of 25 was obtained, although the fitness value was similar to that from reverse calculation with RIGA. It was found that the solution obtained from RIGA showed high reliability compared with that from an exhaustive search.

Then, RC samples of those blendings were actually prepared and tasted by the same 500 consumer panelists whose preference clusters were identified. In this case, each panelist evaluated the

Table 2. Predicting accuracies of FNN models for each cluster

\begin{tabular}{lccc}
\hline & $\mathrm{R}^{2}$ & Maximum absolute error* & Mean absolute error* \\
\hline Cluster 1 & 0.987 & 0.167 & 0.014 \\
Cluster 2 & 0.979 & 0.161 & 0.024 \\
Cluster 3 & 0.994 & 0.106 & 0.014 \\
Cluster 4 & 0.993 & 0.170 & 0.008 \\
\hline Absolute error* $=$ Actual & preference score-preference score calculated by \\
FNN modell
\end{tabular}

Table 3. Validation of optimized blend sample for each cluster.

\begin{tabular}{ccccc}
\hline \multirow{2}{*}{$\begin{array}{c}\text { Reverse estimated sample } \\
\text { (Target cluster) }\end{array}$} & \multicolumn{3}{c}{ Predicted score by FNN-model of each cluster } \\
\cline { 2 - 3 } & Cluster-1 & Cluster-2 & Actual preference score of \\
target cluster & $\mathbf{7 . 0 0}$ & 5.35 & 5.42 & 6.15 \\
Sample 1 (Cluster 1) & 6.39 & $\mathbf{6 . 8 3}$ & 5.83 & $\mathbf{6 . 8 8}$ \\
Sample 2 (Cluster 2) & 6.34 & 6.16 & $\mathbf{6 . 9 2}$ & $\mathbf{6 . 7 8}$ \\
Sample 3 (Cluster 3) & 6.48 & 6.61 & 5.91 & $\mathbf{6 . 9 6}$ \\
Sample 4 (Cluster 4) & & & $\mathbf{7 . 1 0}$ \\
\hline
\end{tabular}

The scores of target clusters are printed in bold type. 
score of one sample, which was prepared as optimum blending in her own cluster, not referring to other samples. This kind of evaluation is not easy, but the average score is reliable since tasting was done by the same 500 panelists. The average preference score in each cluster was calculated and results are shown in Table 3. The prepared sample optimized for each cluster actually showed the highest score compared to the modeling test. Although differences between actual and predicted preference scores (Table 3) were larger than the mean absolute errors of the FNN model (Table 2), differences were small enough compared with the variation range (Table 1). From these results, reverse estimation by RIGA was considered to show sufficient accuracy for practical use.

Our previous paper reported forward FNN modeling and reverse estimation with RIGA relating the blend ratio to sensory scores by skilled coffee tasters (Tominaga et al., 2002b). In the present study, the same approach was carried out for consumer preference data. Appropriate clustering based on consumers' preferences led to good results for highly accurate model construction. Using the forward FNN model thereby constructed, reliable solutions in reverse estimation by RIGA were obtained. Therefore, this approach is considered to be applicable not only for coffee, but also for other products for which preference is an important quality.

After construction of the forward FNN modeling, we are able to obtain fuzzy rules with FNN models, which is a merit not found with ANN modeling. Results of the fuzzy rules agreed well with the preference cluster properties described above. Since understanding of causal reasons for product preference is crucial in marketing research involving identification of consumer characteristics and consumer preference transition, we believe that FNN modeling is very useful for clarifying causal relations on preference.

\section{Conclusions}

Based on RC preferences, consumer panelists at home were divided into four clusters using $12 \mathrm{RC}$ samples. For each preference cluster, FNN models, which can predict preference scores of RC samples, were built using $88 \mathrm{RC}$ samples employing the same panels as the previous clustering test. Model accuracy was very high in each model acquired and mean absolute errors were from $0.8 \%$ to $2.4 \%$. Next, the most preferable blend ratio in each cluster was searched by RIGA in combination of the forward FNN model. According to the optimum blend ratio, RC samples were prepared and tasted by the same panelists to validate sample performance. Those samples actually showed the highest score compared with those from modeling results. This clustering and modeling study was concluded to provide an excellent tool for rapid and efficient development of coffee products.

\section{References}

Furuhashi, T. (1993). Representation of fuzzy rules using neural networks. J. Jpn. Soc. Fuzzy Theory Syst., 5, 204-217.

Hamuro, K. (1997). Consumers' preference prediction system using neural network. Shokuhin Kougyou, 40, 61-70 (in Japanese).

Hanai, T., Katayama, A., Honda, H. and Kobayashi, T. (1997a). Auto- matic fuzzy modeling for Ginjo sake brewing process using fuzzy neural networks. J. Chem. Eng. Jpn., 30, 94-100.

Hanai, T., Ohkusu, E., Honda, H., Ito, F., Sugiura, M., Asano, I. and Kobayashi, T. (1997b). Quality modeling for coffee using knowledge information processing. Nippon Shokuhin Kagaku Kogaku Kaishi, 44, 560-568 (in Japanese).

Hanai, T., Ando, K., Noguchi, H., Honda, H., Takai, C., Furuhashi, T., Uchikawa, Y. and Kobayashi, T. (1998a). Modeling of sensory evaluation for interior tiles using fuzzy neural network. Kagaku Kougaku Ronbunshu, 24, 18-23 (in Japanese).

Hanai, T., Ando, K., Noguchi, H., Honda, H., Takai, C., Kawaide, J. and Kobayashi, T. (1998b). A new selection system for exterior tile using 2 step FNN models. Kagaku Kougaku Ronbunshu, 24, 716721 (in Japanese).

Hanai, T., Noguchi, H., Honda, H., Furuhashi, T., Uchikawa, Y., Kamiya, M., Ishi, T. and Kobayashi, T. (1998c). Quality modeling of Ginjo sake using hierarchical fuzzy neural network. J. Jpn. Soc. Fuzzy Theory Syst., 10, 299-306.

Hanai, T., Iwata, N., Honda, H., Furuhashi, T., Uchikawa, Y. and Kobayashi, T. (1998d). Optimization of koji-making process using FNN and CFGA. Preprints of the 7th International Conf. On Comput. Appli. In Biotech., Osaka, Japan, pp. 215-218.

Hibino, S., Hanai, T., Nagata, E., Matsubara, M., Fukagawa, K., Shirataki, T., Honda, H. and Kobayashi, T. (2001). Fuzzy neural network model for assessment of Alzheimer-type dementia. J. Chem. Eng. Jpn., 34, 936-942.

Honda, H. Hanai, T., Katayama, A., Tohyama, H. and Kobayashi, T. (1998). Temperature control of Ginjo sake mashing process by automatic fuzzy modeling using fuzzy neural networks. J. Ferment. Bioeng., 85, 107-112.

Horikawa, S., Furuhashi, T. and Uchiyama, Y. (1991). A study on fuzzy modeling using fuzzy neural networks. Proc. Int. Fuzzy Eng. Symp, 5, 562-573.

Katahira, H. (1987). "Marketing Science,” Tokyo Univ. Press, Tokyo.

Nagamachi, M. (1992). “Kaiteki Kagaku,” Kaibundo, Tokyo.

Nagamori, E., Honda, H., Hanai, T., Nakanishi, K., Hata, N., Masuda, T. and Kobayashi, T. (2001). Prediction of occurrence of Heterocapsa circularisquama red tide by means of fuzzy neural network. J. Chem. Eng. Jpn., 34, 998-1005.

Nishio, C. (1994). Marketing models by neural network. Oper. Res., 39, 203-208.

Noguchi, H., Hanai, T., Takahashi, W., Ichii, T., Tanikawa, M., Masuoka, S., Honda, H. and Kobayashi, T. (1999). Model construction for quality of beer and brewing process using FNN. Kagaku Kogaku Ronbunshu, 25, 695-701 (in Japanese).

Noguchi, H., Hanai, T., Honda, H., Harrison, L.C. and Kobayashi, T. (2001). Fuzzy neural network-based prediction of motif for MHC class II binding peptides. J. Biosci. Bioeng., 92, 227-231.

Sagara, Y. (1998). Sensing of food preference and food sensitivity engineering. Kagaku to Seibutsu, 36, 287-289 (in Japanese).

Tomida, S., Hanai, T., Ueda, N., Honda, H. and Kobayashi, T. (1999). Construction of COD simulation model for activated sludge process by fuzzy neural network. J. Biosci. Bioeng., 88, 215-220.

Tomida, S., Hanai, T., Honda, H. and Kobayashi, T. (2001). Construction of COD simulation model for activated sludge process by recursive fuzzy neural network. J. Chem. Eng. Jpn., 34, 369-375.

Tominaga, O., Ito, F., Hanai, T., Honda, H. and Kobayashi, T. (2002a). Sensory modeling of coffee with a fuzzy neural network. J. Food Sci., 67, 363-368.

Tominaga, O., Ito, F., Hanai, T., Honda, H. and Kobayashi, T. (2002b). Determination of the blending ratio of regular coffee samples by information technology. J. Chem. Eng. Jpn., 35, 137-143.

Yoshikawa, H., Hanai, T., Tomida, S., Honda, H. and Kobayashi, T. (2001). Determination of operating conditions in activated sludge process using fuzzy neural network and genetic algorithm. J. Chem. Eng. Jpn., 34, 1033-1039. 